\title{
DIÁLISIS PERITONEAL
}

\section{Correlación entre volumen, transporte peritoneal e hipertensión arterial}

Orlando Olivares, $M D^{*}$, Sandra Juliana Jiménez, MD* Sandra Rubiano,**, Mauricio Ruiz, MD***, Carlos Roselli, MD***, Francisco Barreto, $M D^{* * * *}$, Rodolfo Torres, $M D^{* * *}$

\section{Resumen}

Objetivo: determinar si existe relación entre el volumen medido por bioimpedanciometría, el tipo de transporte peritoneal y la incidencia de hipertensión arterial (HA) en pacientes en diálisis peritoneal (DP).

Materiales y métodos: se realizó un estudio de corte transversal. Se incluyeron 72 pacientes en DP del Hospital de San José a quienes se les midió el volumen de líquido corporal mediante bioimpedanciometría. Los pacientes fueron clasificados según las características del transporte peritoneal, establecido a través de la prueba de equilibrio peritoneal (PEP), en transportadores altos (TA), promedio (TP) y bajos (TB). Se evaluaron las cifras de tensión arterial, clasificando a los pacientes en normotensos, hipertensos controlados con hipotensores e hipertensos no controlados.

Resultados: el exceso de agua fue de 2,98 +/- 2,36 L en TP y de 3,631L +/- 1,81 L en TA. En los pacientes normotensos, hipertensos controlados con medicación e hipertensos no controlados, se encontraron valores promedio de exceso de agua 3,16 $\mathrm{L}+/$ - 2,56, 2,79 L +/- 2,11 y 3,79 L +/- 2,39 L respectivamente. Se halló una frecuencia de normotensión de 28,1\%, hipertensión controlada de 56,1\% y de hipertensión no controlada de 15,8\%. en los TP. En los TA estos valores fueron $10 \%, 50 \%$ y $40 \%$ respectivamente.

Conclusión: los TA tienden hacia un mayor exceso de líquido que los TPy tienen mayor frecuencia de HA no controlada. Esto podría explicar el exceso de mortalidad reportado en este grupo. El exceso de volumen independientemente del estado transportador, se relaciona con la presencia de $\mathrm{HA}$.

Palabras clave: diálisis peritoneal, hipertensión arterial, volumen de líquido corporal, transporte peritoneal alto.

\section{Introducción}

La experiencia acumulada y el progreso en las técnicas dialíticas han permitido que la diálisis peritoneal (DP) se convierta en una opción de terapia de reemplazo renal crónico comparable con la hemodiálisis ${ }^{1}$ (HD).

Los reportes iniciales de bajas tasas de sobrevida con DP, se asociaron con alta incidencia de peritonitis y de complicaciones relacionadas con disfunción del catéter. Sin embargo, con la mejoría en las técnicas de conexión y la introducción de nuevos catéteres al mercado, se ha logrado disminuir el riesgo de disfunción de

Residente II de Nefrología, Fundación Universitaria de Ciencias de la Salud.

** Nutricionista Unidad Renal, Hospital de San José.

*** Instructor Asistente Fundación Universitaria de Ciencias de la Salud. Hospital de San José

*****Profesor Asistente Fundación Universitaria de Ciencias de la Salud, Hospital de San José. éste y las complicaciones infecciosas. Dichos avances han permitido que del $15 \%$ al $20 \%$ de la población en diálisis se encuentre en este tipo de terapia. ${ }^{1}$

Varios estudios a corto y mediano plazo señalan una tasa de supervivencia similar en HD y DP a pesar de ello, la DP tiene un tiempo de duración limitado a largo plazo. ${ }^{2}$

Varios factores podrían estar involucrados en la falla de la técnica y la mortalidad de los pacientes en DP, en particular la enfermedad cardiovascular, ${ }^{3}$ la pérdida de la función renal residual y el deterioro del estado nutricional. Existe fuerte evidencia de que el estado de transporte peritoneal, valorado por el transporte de solutos de bajo peso molecular ${ }^{4}$ y la capacidad de ultrafiltración ${ }^{5}$ influyen sobre el pronóstico, tanto en relación con falla de la técnica, como en la mortalidad de origen cardiovascular. 
De gran interés han sido los reportes acerca de cómo las características del transporte peritoneal tienen impacto significativo sobre el pronóstico. El subanálisis del estudio CANUSA (estudio cooperativo acerca de las características de los pacientes en diálisis en Estados Unidos y Canadá) evidenció mayor tasa de mortalidad y morbilidad en los pacientes TA (mayor valor del cociente creatinina del dializado / creatinina del plasma). ${ }^{6}$ Es posible que los resultados tengan que ver con la pérdida de ultraflitración, incremento en la absorción de glucosa y la mayor pérdida de albúmina a través del dializado que caracteriza a estos pacientes.

La mayor prevalencia de enfermedad cardiovascular e hipertensión en los pacientes anúricos y con mayores cocientes de creatinina del dializado/plasma, sugiere que el inadecuado manejo del estado hídrico contribuye al mal pronóstico y pobre supervivencia de la técnica de DP. De la misma forma, estos hallazgos podrían explicar los reportes de estudios recientes, que evidencian mayor prevalencia de HA e hipertrofia ventricular izquierda ${ }^{7}$ en los pacientes en DP comparados con aquellos en HD.

Muy pocos estudios han evaluado la relación entre el estado del volumen intravascular, las características del transporte peritoneal, la función renal residual y la incidencia de HA en pacientes en DP.

Por medio del presente estudio queremos analizar si existe relación entre el estado del volumen medido por bioimpedanciometría, el tipo de transporte peritoneal y la prevalencia de HA en pacientes en DP.

\section{Materiales y métodos}

\section{Protocolo}

Este estudio de corte transversal se realizó en el período comprendido entre julio y noviembre de 2003 e incluye a los pacientes en programa de DP del hospital de San José. A la población estudiada se le realizó valoración del volumen de líquido corporal por medio de bioimpedanciometría, se clasificaron los pacientes según las características del transporte peritoneal, establecido a través de la prueba de equilibrio peritoneal (PEP) en TA, TP y TB y se evaluaron las cifras de tensión arterial, calificándolos como normotensos, hipertensos controlados con medicamentos e hipertensos no controlados.
Se incluyeron todos aquellos sin criterios de exclusión como la presencia de peritonitis dentro de los tres meses anteriores al ingreso del estudio, insuficiencia cardiaca congestiva estadío IV según la clasificación de la NYHA, infección activa y presencia de malignidad.

\section{Pacientes}

En este estudio de corte transversal, 72 enfermos en programa crónico de DP llenaron el único criterio de inclusión: insuficiencia renal crónica avanzada y que al menos hubiesen estado dos meses en programa de DP.

\section{Prueba de equilibrio peritoneal}

Las características de la membrana peritoneal en cuanto al tipo de transportador fueron caracterizadas usando la PEP, que es una prueba estandarizada en la cual, después de drenar el líquido de permanencia nocturna se instilan dos litros de solución de diálisis peritoneal con dextrosa al $2.5 \%$ en la cavidad peritoneal (tiempo cero) y se dejan durante cuatro horas. La creatinina, úrea y glucosa del líquido de diálisis se miden en el tiempo cero, a las dos y a las cuatro horas de permanencia y se determinan los valores séricos a las dos horas. A la cuarta hora se drena el líquido del dializado de la cavidad peritoneal midiéndose el volumen del mismo. Para cada uno de esos tiempos de permanencia, se coloca la proporción dializado/plasma (DP) de creatinina y úrea, así como la proporción de glucosa del líquido peritoneal al tiempo de drenaje con respecto a su concentración al iniciar la infusión (D/DO). Con base en los resultados los pacientes se clasifican como TA (D/P de creatinina $<0,8)$ TP (D/P creatinina entre $0,5-0,8)$ y TB (DP de creatinina $<0,5)$. La PEP en nuestro estudio se realizó entre uno y tres meses antes de efectuar el estudio de bioimpedanciometría. Estos resultados retrospectivos del PEP no alteran la naturaleza transversal del estudio, en vista de que las características de la membrana peritoneal en condiciones normales tampoco se alteran en este lapso.

\section{Función renal residual}

La función renal residual fue evaluada al mismo tiempo que la PEP de cuatro horas. Los cálculos se realizaron tomando el promedio de la sumatoria del nitrógeno ureico y la depuración de creatinina en la orina de 24 horas. 
Los datos fueron obtenidos de la historia clínica de los pacientes en DP.

\section{Estado de hipertensión arterial y numero de hipotensores}

El estado de las cifras tensionales fue obtenido del último control histórico registrado en la consulta médica y de enfermería de los pacientes en DP. La valoración de las cifras tensionales se realizó el mismo día de la bioimpedanciometría. Se definió como HA la presencia de cifras tensionales mayores de 140/90 mm Hg en tres tomas consecutivas. De acuerdo con ello fueron clasificados como normotensos aquellos con cifras menores de 140/90 mm Hg, hipertensos controlados cuando los valores fueron menores de 140/90 mm Hg con la administración de medicamentos antihipertensivos e hipertensos los que cursaron con cifras mayores de 140/ $90 \mathrm{~mm} \mathrm{Hg}$.

El número de hipotensores se tomó del último control histórico de la consulta anterior a la inclusión del paciente al estudio.

\section{Estado de volumen}

Fue valorado mediante bioimpedanciometría de frecuencia múltiple, medida a frecuencias espaciadas logarítmicamente entre $5 \mathrm{Khz}$.(tecnología Xitron). Se colocaron electrodos pares en el dorso de la mano derecha y el pie, con el electrodo inyector de corriente en situación distal. El líquido peritoneal fue drenado de la cavidad antes de la realización del examen.

El análisis de la medida del volumen extracelular y el exceso de agua fueron obtenidos a partir de los datos analizados por el software (xitron). ${ }^{9} \mathrm{El}$ exceso de agua fue definido como el valor estimado por el bioimpedanciómetro de la cantidad de agua por encima del peso seco verdadero.

\section{Análisis de los resultados}

Inicialmente se realizó uno descriptivo de todas las variables medidas. A las categóricas de tipo nominal se les calcularon las proporciones de ocurrencia y a las numéricas la media, medianas y desviaciones estándar. Para establecer las diferencias entre los resultados de variables numéricas entre los grupos normotensos e hipertensos, se utilizó la prueba t de student y para establecer las diferencias de acuerdo con el tipo de transportador, se agruparon los resultados y se hizo un análisis de subgrupos.

Estos datos fueron estudiados en tablas $2 \times 2$ y para determinar asociaciones se utilizóla prueba de Chi cuadrado o la prueba exacta de Fisher cuando el número esperado por celda fue menor o igual a 5. Además se calcularon los riesgos (RR y OR) con sus respectivos intervalos de confianza del $95 \%$. El nivel de significancia para las diferentes pruebas estadísticas se estableció en 0,05.

Para el manejo de los datos y el análisis de la información se utilizó el paquete estadístico SPSS versión 8.0.

\section{Consideraciones éticas}

El estudio se presentó ante el comité de ética en investigaciones de la Fundación Universitaria de Ciencias de la Salud, de acuerdo con lo establecido en la resolución 8430 de 1993 ("Normas científicas y administrativas para la investigación en salud") del Ministerio de Salud y fue clasificado por el comité como investigación "con riesgo mínimo".

El estudio sigue los lineamientos jurídicos y éticos del país y también aquellos contemplados en la última modificación (Edimburgo, Escocia, octubre de 2000), de la declaración de Helsinki de la asociación médica mundial ("Principios éticos para la investigación que involucra sujetos humanos").

La participación en la investigación fue voluntaria y la aceptación se realizó luego de brindarles información precisa. Los objetivos, alcances y resultados del estudio serán conocidos por los participantes y por la comunidad académica. Los datos y registros obtenidos se consignaron de tal forma que se protegiera la confidencialidad de los sujetos.

\section{Resultados}

De los 120 pacientes vinculados al programa DP del hospital de San José, 72 cumplieron con los criterios de inclusión (Tabla 1). 
Teniendo en cuenta que los TB eran solo cinco pacientes y que desde el punto de vista fisiopatológico no constituían el principal grupo de riesgo para el desarrollo de sobrecarga de volumen, se excluyeron del análisis estadístico y sólo se tomaron en cuenta los pacientes TPy TA.

\begin{tabular}{lc}
\multicolumn{2}{c}{ Tabla I. Características demográficas } \\
\multicolumn{1}{c}{$\begin{array}{c}\text { Características de los } \\
\text { pacientes (promedio) }\end{array}$} \\
\hline EDAD & 53,5 \\
\hline SEXO & \\
Masculino & $58,3 \%$ \\
Femenino & $41,6 \%$ \\
\hline CAUSA DE FALLA RENAL & \\
Diabetes & $26 \%$ \\
Hipertensión arterial & $6,3 \%$ \\
Enfermedad glomerular & $26 \%$ \\
Enfermedad autoinmune & $8,3 \%$ \\
Uropatía obstructiva & $2,7 \%$ \\
Enfermedad poliquística & $2,7 \%$ \\
Otras & $26 \%$ \\
\hline TIPO DE TRANSPORTADOR & \\
Bajo & $5 \%$ \\
Promedio & $79 \%$ \\
Alto & $15 \%$ \\
\hline FUNCION RENAL RESIDUAL & \\
<2ml/min & $81,94 \%$ \\
>2ml/min & $18,06 \%$ \\
\hline CIFRAS DE TENSIÓN ARTERIAL \\
Normotensos & $22,2 \%$ \\
Normotensos con hipotensores & $55,5 \%$ \\
Hipertensos & $22,2 \%$ \\
\hline TIPO DE TRANSPORTADOR & \\
CAPD & \\
APD & $11,11 \%$ \\
\hline
\end{tabular}

La relación entre el exceso de agua y el tipo de transporte peritoneal, fue de 2,98 L +/- 2,36 en los TP y de 3,63 L +/-1,81 en TA (p=0,408). Se realizó un subanálisis para definir si en el grupo de los TP existía relación entre el exceso de agua y la función renal residual, sin encontrarla.

Se halló un aumento en la frecuencia de pacientes con exceso de agua entre los TA cuando se realizaron análisis comparando subgrupos con excesos de agua mayores de 1, 2 y 3 L (Tabla 2).

Al clasificar los pacientes en normotensos, hipertensos controlados e hipertensos no controlados, se encontraron valores promedios de exceso de agua de 3,16 L +/- 2,56, 2,79 L +/- 2,11 y 3,79 L +/- 2,39 respectivamente (Kruskal Wallis $p=0,262$ ).

Al unir los pacientes normotensos y normotensos con medicación antihipertensiva en un solo grupo y compararlos con el grupo de hipertensos no controlados, se encontraron valores promedios de exceso de agua de $2,91 \mathrm{~L}+/-2,25$ y $3,78 \mathrm{~L}+/-2,39(p=0,21)$ respectivamente (Tabla 3).

El análisis de la presencia de hipertensión no controlada en los grupos de pacientes que tenían exceso de agua, evidenció una tendencia a encontrar un mayor número de hipertensos en aquellos que tenían excesos mayores de 1 y 3 L cuando se los comparó con los que tenían excesos menores a estos valores. Esta tendencia no se observó cuando se compararon los que tenían excesos mayores de $2 \mathrm{~L}$ de agua, con los que tenían excesos menores de este valor (Tabla 4).

En cuanto a la relación entre el tipo de transporte peritoneal y las cifras tensionales, se encontró una frecuencia de normotensión en el grupo TP de $28,1 \%$, hipertensos controlados con hipotensores de $56,1 \%$ y

Tabla 2 Relación entre el tipo de transporte peritoneal y el exceso de agua

TIPO DE TRANSPORTE PERITONEAL

\begin{tabular}{lccc} 
& Promedio $(n=57)$ & Alto $(n=10)$ & $p$ \\
\hline Exceso de agua $>$ I L & $42(37 \%)$ & $9(80 \%)$ & NS \\
Exceso de agua $>$ 2 L & $38(66,7 \%)$ & $8(80 \%)$ & NS \\
Exceso de agua $>$ 3 L & $31(54,4 \%)$ & $7(70 \%)$ & NS
\end{tabular}


de hipertensión no controlada de $15,8 \%$. En el grupo de TA estos valores fueron de 10,50 y $40 \%$ respectivamente. Al unir los pacientes normotensos con y sin medicación antihipertensiva y compararlos con los pacientes hipertensos se sugirió una mayor tendencia de pacientes hipertensos no controlados en los TA (40\%) con respecto a los $\operatorname{TP}(15 \%)(p=0,093)$.

Se observó una posible asociación entre la ausencia de función renal residual y el tiempo en diálisis peritoneal (Tabla 5).

No encontramos asociación entre el tiempo de permanencia de los pacientes en diálisis con la presencia de hipertensión arterial no controlada (Tabla 6), como tampoco se observó entre la ausencia de función renal residual y el exceso de agua (Tabla 7).

\section{Discusión}

La etiología de la falla renal en el grupo de pacientes analizados difiere un poco de la reportada en la literatu-

Tabla 3. Relación entre el exceso de agua con la presión arterial.

\begin{tabular}{lccc} 
& $\begin{array}{c}\text { Pacientes } \\
\text { normotensos } \\
\text { (con y sin } \\
\text { medicación)(54) }\end{array}$ & $\begin{array}{c}\text { Pacientes } \\
\text { hipertensos } \\
(13)\end{array}$ & P \\
\hline $\begin{array}{l}\text { Exceso de } \\
\text { agua (litros) }\end{array}$ & 2,21 & 3,71 & 0,21
\end{tabular}

Tabla 4. Relación entre normo e hipertensión con el exceso de agua mayor vs. menor de 1,2 y 3 litros.

Pacientes normotensos

\begin{tabular}{ll}
$\begin{array}{l}\text { Exceso de } \\
\text { agua }\end{array}$ & $\begin{array}{c}\text { (con y sin } \\
\text { medicación)(54) }\end{array}$ \\
\hline
\end{tabular}$$
>1
$$$$
<
$$$$
>
$$$$
<2
$$$$
>3
$$$$
<3
$$

$21,6 \%$

$12,5 \%$

$19,6 \%$

$80,4 \%$

$19,0 \%$

$81,0 \%$

$23,7 \%$

$76,3 \%$

$13,8 \%$
Pacientes

ra, ya que contrario a otras series, tanto la diabetes como la enfermedad glomerular primaria son las causas principales de la insuficiencia renal terminal ${ }^{10}$; esto se debe a que la glomerulonefritis ocurre principalmente en personas jóvenes, grupo etáreo que escoge con mayor frecuencia vincularse a esta terapia. Por el contrario, los pacientes con nefropatía diabética tienen mayor edad y más comorbilidades, entre las que se cuentan alteraciones visuales incapacitantes que hacen que estos pacientes dependan de otras personas para su cuidado y escojan vincularse a los programas de hemodiálisis. Así mismo, la falla renal terminal de etiología desconocida es prevalente, debido a que en muchos casos los pacientes consultan en forma tardía y no se logra realizar diagnóstico etiológico.

En nuestro grupo de análisis, el 77,7\% de los pacientes fueron hipertensos controlados con medicamentos o no

\section{Tabla 5. Relación entre la función renal residual y el tiempo en dialisis peritoneal}

\begin{tabular}{lccc}
$\begin{array}{l}\text { Tiempo } \\
\text { en diálisis }\end{array}$ & $\begin{array}{c}\text { Función renal residual } \\
<\mathbf{2} \mathbf{~ m l} / \mathbf{m i n}\end{array}$ & $>\mathbf{2} \mathbf{~ m l} / \mathbf{m i n}$ & $\boldsymbol{P}$ \\
\hline$<$ I año & $57,1 \%$ & $42,9 \%$ & 0,009 \\
I-5 años & $84 \%$ & $15 \%$ & 0,005 \\
$>5$ años & $100 \%$ & $0 \%$ & 0,003
\end{tabular}

Tabla 6. Relación entre tiempo de permanencia en diálisis e hipertensión no controlada.

\begin{tabular}{|c|c|c|c|}
\hline $\begin{array}{l}\text { Tiempo } \\
\text { en diálisis }\end{array}$ & $\begin{array}{c}\text { Pacientes } \\
\text { hipertensos } \\
\text { no controlados } \\
\text { (13 pacientes) }\end{array}$ & Porcentaje & $p$ \\
\hline$<$ de laño & $5 / 13$ & $38,5 \%$ & \\
\hline Entre $1-5$ años & $5 / 13$ & $38,5 \%$ & \\
\hline$>1$ año & $3 / 13$ & $23,1 \%$ & \\
\hline Total & & $100 \%$ & \\
\hline
\end{tabular}

Tabla 7. Relación entre exceso de agua y la función renal residual.
Función renal residual

$<2 \mathrm{ml} / \mathrm{min}>2 \mathrm{ml} / \mathrm{min} p$

\section{Exceso de}

agua

2,71

3,61

0,26 
controlados a pesar del uso de múltiples fármacos. Las causas de la HA pueden ser multifactoriales; sin embargo, el exceso de volumen puede jugar un papel importante. Esto se refleja en el hecho de que el grupo de hipertensos no controlados presentaba un exceso de volumen de casi un litro, en comparación con los grupos normotensos con y sin medicación. Los resultados están en concordancia con lo reportado por Kooning, ${ }^{11}$ quien encuentra mayor exceso de volumen de agua en los grupos de pacientes hipertensos y aquellos con presencia de sobrecarga ventricular. Aunque los hallazgos de nuestro estudio muestran diferencias significativas desde el punto de vista estadístico en cuanto a la presencia de exceso de volumen entre los grupos normotensos e hipertensos, sí se encontró una tendencia acerca de mayor exceso de líquido en los hipertensos, en especial en el grupo de los no controlados. Como lo menciona la literatura, la optimización del volumen hídrico de los diferentes compartimientos corporales puede ser un factor de buen pronóstico en los pacientes en diálisis peritoneal, por la dismunución del riesgo cardiovascular inherente al pobre control de las cifras tensionales relacionado con el exceso de volumen ${ }^{12}$.

Fue paradójico que al comparar a los pacientes normotensos sin medicación con los hipertensos controlados, se encontró un balance positivo de $600 \mathrm{cc}$ en el primer grupo. Creemos que podría explicarse por el mayor número de enfermos con grados leves a moderados de insuficiencia cardíaca entre los normotensos, lo cual haría que no se presentara hipertensión a pesar del exceso de líquidos.

Se sugirió una relación entre la presencia de exceso de agua y el estado de transporte del peritoneo, ya que cuando se compararon los grupos de TP y TA, éstos últimos tuvieron tendencia a presentar exceso de volumen hídrico en comparación con los primeros; además, los TA presentaron con mayor frecuencia HA controlada y no controlada. A pesar de la débil correlación, estas observaciones sugieren que el estado de transporte alto de la membrana peritoneal (relación D/P alta) es un factor de riesgo para la sobrehidratación progresiva de los pacientes en diálisis peritoneal. Estos hallazgos están de acuerdo con el trabajo de Constantijn ${ }^{13}$ y podrían explicar la mayor tasa de mortalidad cardiovascular reportada en la literatura en este grupo. ${ }^{14-15}$
No surgió relación entre la pérdida de la función renal residual y el exceso de agua, a diferencia de lo reportado por la literatura. Esto pudo deberse a una posible inconsistencia en la recolección de orina de 24 horas para la evaluación de la función renal residual, debido a incumplimiento por parte de los pacientes de las recomendaciones dadas. Estas observaciones no demeritan la importancia de la función renal residual, como factor que determina el estado hídrico y el pronóstico en los pacientes en diálisis peritoneal. ${ }^{16}$

Encontramos una posible asociación entre el tiempo de duración en diálisis y la pérdida de la función renal residual, lo cual está acorde con lo encontrado por otros grupos. ${ }^{17}$ Aunque la naturaleza del estudio no permite determinar la tasa/mes de pérdida de la función renal residual, es interesante notar la ausencia de ésta en el grupo de pacientes con más de cinco años en el programa de DP. Siendo la pérdida de la función renal residual uno de los principales factores negativos en cuanto a la supervivencia de los pacientes, las intervenciones que favorezcan la menor tasa de pérdida de ésta podrían traducirse en mejoría del pronóstico a largo plazo.

Existen varias limitaciones del estudio: el pequeño tamaño de la muestra que no permitió que los resultados fueran estadísticamente significativos a pesar de que se encontraron tendencias claras para los puntos examinados. Su naturaleza transversal no permite plantear conclusiones acerca del pronóstico a largo plazo de los pacientes, y por último, debido a que no se realizó seguimiento, no pudimos establecer factores de riesgo para el aumento de la mortalidad. De la misma forma, tampoco se realizó control del cumplimiento de la prescripción dialítica ni dietaria por parte de los pacientes.

Los resultados de éste estudio deben considerarse a la luz de sus limitaciones y deberán compararse con otro prospectivo que incluya un número mayor de pacientes en DP.

\section{Conclusión}

Los pacientes con transporte peritoneal alto parecen tener un exceso de líquido mayor que los pacientes TP; además, parecen presentar con más frecuencia HA no 
controlada, lo cual podría explicar la alta mortalidad reportada en trabajos anteriores. El exceso de volumen, independiente del estado transportador, se relaciona con la presencia de HA.

Estos hallazgos denotan la importancia del control adecuado de líquidos para disminuir la incidencia de HA y por tanto la mortalidad cardiovascular de los pacientes en DP.

\section{Agradecimiento}

Agradecemos la colaboración de FRESENIUS MEDICAL CARE COLOMBIA por su soporte y ayuda para la realización de esta investigación.

\section{Bibliografía}

1. Gokal. Is lorg-term technique survival on continouos ambulatory peritoneal dialysis possible. Perit Dial Int 1996;16 :553-55.

2. Maiorca R.A multicenter selection adjusted comparison of patient and technique survivals in CAPD and hemodialysis. Perit Dial Int 1991; 11:118-127.

3. Lameire N. Cardiovascular risk factors and their management in patients on continuous ambulatory peritoneal dialysis. Kidney Int 1994; 46:S31-S38 (supp48).

4. Churchill DN. Increased peritoneal membrane transport is associated with decreased CAPD technique and patient survival. J Am SocNephrol 1997: 8: 189A
5. Davies SJ, Phillips L, Naish PF, Russell GI. Peritoneal ultrafiltration and survival in CAPD patients. J Am Soc Nephrol 1997; 8: 263A (abstr)

6. Churchill DN. CAPD patient and 1 technique survival is worse increased membrane permeability. Perit Dial Int. 1996;16(s2):21.

7. Guiseppe E. Long-term patients CAPD are volumen expanded and presented and display more severe hypertrophy ventricular left than hemodialysis patients. Nephrol Dial Transplant 2001,16:1459-62.

8. Twardowski. Clinical value of standardized equilibration test in CAPD patients. Blood Purif 1989;7: 95-108.

9. Woodout. Measurement of total body water by bioelectrical impedance in chronic renal failure. Eur J Clinic Nutr 1996;50.676-82.

11. Konings. Fluid status, blood pressure and cardiovascular abnormalities in patients on dialysis peritoneal. Perit dial Int. 2002;22: 477-87.

12. Cheigh. How well is hypertension controlled in CAPD patients? Adv Perit Dial 1994; 10: 55-8.

13. Constantijn. Fluid status in patients CAPD is related to peritoneal transport and residual renal function. Nephrol Dial Transplant. 2003;18:797-803.

14. Canusa. Peritoneal Dialysis study group. Adequacy of dialysis and nutrition in continuous peritoneal dialysis: association with clinical outcome. J Am Soc Nephrol 1996;7198-207.

15. Tao W. Increased peritoneal permeability is associated with decreased fluid and small solute removal and higher mortality in CAPD patients. Nephol Dial Transplant 1998; 13: $1242-49$.

16. Cheung-Chun S. Are peritoneal dialysis patients with and without residual renal function equivalent for survival study?. Nephrol Dial Transplant 2003; 18: 990-95.

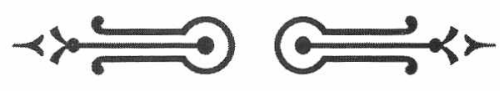

